\section{The 26th annual conference of the British Association for Immediate Care, Birmingham NEC, 14-16 November 2003}

\section{INTRODUCTION}

\author{
P. E. Olliffe. GP Clinical Fellow, Cardiff
}

Birmingham 2003 was my first experience of a BASICS conference. Being relatively new to the field of pre-hospital care, and a new member of BASICS, I was apprehensive, but my positive experiences on the Immediate Care Course reassured me. The BASICS conference far exceeded my expectations and I would recommend it to new and established members alike. The comprehensive and varied programme catered for everyone and held our interest throughout the long days. Particularly informative were the presentations highlighting new areas of research and development in immediate care medicine, resuscitation and car safety devices (or should that be "rescuer injury inflictors") Workshops provided an opportunity to learn new skills and use new pieces of equipment, as well as providing a forum for debating contentious issues and management dilemmas. For those with the spare cash, and a passion for gadgets, the frequent coffee breaks provided the opportunity to peruse the trade stands for the latest in medical equipment. Overall, the pace was lively and the discussions provoked were doubly so. It was the three opening presentations ("Pre-hospital care in Malta", "Heat illness - experience from the Gulf" and "BASICS in the Arctic ${ }^{\prime \prime}$ ) however, that really set the atmosphere for the conference. They encapsulated, for me, the spirit of BASICS itself: friendship and generosity; an eagerness to share and to facilitate learning; a striving for achievement and a hunger for adventure and new horizons. I certainly found all this and more in the people that welcomed me so warmly into an organisation that I am looking forward to being a part of.

\section{Free paper abstracts}

\section{PREHOSPITAL USE OF LARYNGEAL MASK AIRWAYS IN THE WARWICKSHIRE AMBULANCE SERVICE: TWO YEARS' EXPERIENCE}

K. Pattinson*, I. Todd, J. Thomas, M. Wyse. *University Hospitals NHS Trust Coventry; Warwickshire and Warwickshire Ambulance Service

The Laryngeal mask airway (LMA) is a safe and popular method of airway control. Its efficacy in the pre-hospital environment has led to its use by ambulance services worldwide. We describe the experiences of the first two years of LMA use in this ambulance service. We believe we were the first service in the UK to issue LMAs to ambulance technicians. Methods: 1) Every staff member attended a standard Intavent LMA training session. 2) LMAs were introduced into practice, in April 2001 for paramedics and October 2001 for technicians. 3) Survey performed in April 2003, of all clinical staff and summarised below. 4) We analysed the computerised records database of all patients who had LMAs inserted during the study period. Results: 134 surveys received (85\% response). $6.5 \%$ staff used LMA $>6$ times, 35.9\% 3-5 times, $56.5 \% 1-2$ times, $1.1 \%$ never. $69(51.5 \%)$ had additional theatre training, all found the experience very useful. Oral intubation was still considered the first choice for airway care in $78 \%$ of responses (LMA $2 \%$, no response $20 \%$ ). Most would see LMA as great loss if removed from practice. Analysis of case records: 70 attempted placements - 61 successful (87\%), 2 unsuccessful, 7 not recorded. Conditions: Cardiac Arrest, Head injury, overdose, trauma. 9 successful insertions subsequently replaced by endotracheal tube prehospital. 29 LMAs inserted after unsuccessful endotracheal intubation, all these cases LMA was successfully inserted. Conclusions: Most ambulance staff have used the LMA on few occasions. A success rate of $87 \%$ is similar to that described by other studies in non-physicians in the emergency situation. It is of concern that successful LMA placements are being removed in order to achieve endotracheal intubation. There needs to be improved education in LMA use and the current training could probably be improved.

\section{IS THE "GOLDEN HOUR" A PREHOSPITAL EVENT?}

R. Mackenzie, D. Beavan. Mid Anglia General Practitioner Accident Service (MAGPAS)

Two historical concepts underpin trauma system development. The first is that there is a tri-modal distribution of trauma deaths; the majority occurring in the first hour (immediate), approximately 30\% occurring within the first few hours (early) and a much smaller proportion occurring after days to weeks (late). The second is that there is a 'golden hour' window of opportunity in which early and late deaths can be prevented by aggressive resuscitation and surgical intervention. The emphasis placed on these concepts has led to the introduction of the 'Platinum Ten Minutes' as a maximum on-scene time for pre-hospital immediate care practitioners.

Road traffic related incidents are the commonest cause of major trauma in the UK. Although there is evidence that increases in total prehospital time are associated with increased mortality, there is little evidence to support a tri-modal distribution, and thus the concept of preventable deaths through hospital intervention within the golden hour. The distribution of road traffic related deaths in the UK is exponential and the mean pre-hospital time is 59 minutes (SD 42 minutes, from 16,770 patients with major trauma following road traffic related incidents and held on the UK Trauma Audit and Research Network database). In a study of 329 road traffic related deaths within Cambridgehsire between 1997 and 2001, the mean pre-hospital time was 57 minutes (SD 25 minutes, $n=95$ ).

While there is no doubt that time remains a critical factor in trauma care, appropriate interventions in the pre-hospital phase are currently discouraged in an effort to save time. The golden hour is a pre-hospital event! If there is any window of opportunity, it is to develop trauma systems such that the pre-hospital phase can be used for active meaningful intervention related to the patient's immediate needs without unnecessarily delaying their transfer to hospital.

004 A FIELD DECISION TOOL TO GUIDE APPROPRIATE USE OF HELICOPTERS TO TRANSPORT TRAUMA PATIENTS FROM INCIDENT SCENE TO HOSPITAL

J. J. M. Black, M. E. Ward, D. J. Lockey*. Emergency Department and Department of Anasesthesia and Intensive Care, John Radcliffe Hospital, Oxford; *Frenchay Hospital, Bristol

The clinical priorities for injured patients at the scene are identical to those in the emergency department resuscitation room. We present a simple algorithm for field use as a guide to immediate care doctors and ambulance services attending a trauma patient in the field as to the most appropriate mode of transport from the incident scene to hospital. This has been developed after reviewing the literature and reflects the operational experience of the authors delivering pre-hospital critical care.

The decision to use a helicopter for transport, although initially attractive, is not always straight forward, and depends on a number of important factors: aircraft availability, the environmental conditions, the physiological status of the patient and presence of specific injury patterns, incident scene accessibility, aircraft configuration and clinical skills of the crew, and the proximity and resources of adjacent hospitals. Placement of a seriously injured patient in a helicopter without critical care interventions may be hazardous, and may expose them to the risk of secondary injury - under such circumstances transport by road may be the safer option.

Critically injured patients, who potentially have the most to gain by rapid transport to multidisciplinary definitive care, may not be safely transported to hospital by helicopter unless there are the resources for prehospital critical care at the scene. This raises important questions on how prehospital care transport systems could evolve and be potentially integrated into regionally based trauma centres. Our algorithm, which we believe to be widely applicable, considers all of these variables, and 
attempts to guide this complex decision making in a logical and systematic way.

\section{IMMEDIATE CARE SCHEME PROVISION OF SPECIALIST MEDICAL SUPPORT TO THE AMBULANCE SERVICE: THE MAGPAS EMERGENCY MEDICAL TEAM}

S. J. Lewis, N. Church, R. Mackenzie. Mid Anglia General Practitioner Accident Service (MAGPAS)

A review of the activity of a rural Immediate Care Scheme (ICS) in 2000 revealed that reliance on volunteer availability and a wide variation in doctors' ability to meet the needs of seriously injured patients resulted in an unpredictable and highly variable service. The development and evaluation of a County-wide Emergency Medical Team (EMT) involving a volunteer doctor and paramedic was proposed as an alternative to the traditional ICS structure. The key principles of the EMT concept were (1) achieving and maintaining a consistently high standard of competence across the spectrum of ICS activity; (2) encouraging volunteers to schedule their ICS activity within a Personal Development Plan; and (3) achieving the aims of the ICS and maintaining clinical governance.

A 24 day training course covering ten core competency themes Operational Environment, Resuscitation and Clinical Care, Equipment and Monitoring, Care of Children, Rescue and Extrication, Analgesia and Sedation, Pre-hospital Emergency Anaesthesia, Special Incidents Major Incidents and Retrieval and Transfer) was developed and delivered over five months. The course involved 50 hours of lectures, 94 hours of practical sessions, 11 hours of discussion and 6 hours of formal assessment. High fidelity human simulators were used to provide training and assessment in pre-hospital critical care interventions and problems. The EMT became active on 2 June 2003, providing cover for an average of 20 days a month between 07:00 and 19:00. In the first three months of operations, the EMT were requested to attend 204 incidents. Compared to the same period in 2002, the EMT were mobilised to a higher proportion of incidents than conventional ICS doctors. The EMT will be evaluated for a minimum of 1 year within the context of a case control study of the impact of a specialist pre-hospital immediate medical care service on mortality and morbidity following major trauma.

\section{THE USE OF METHYLPREDNISOLONE IN THE TREATMENT OF TRAUMATIC SPINAL CORD INJURY}

K. Crewdson. Guys, Kings and St Thomas' School of Medicine, London

The annual incidence of spinal cord injury in the UK is 10 to 15 per million of the population. Events occurring within the cord following traumatic injury exist as two distinct phases. Primary injury is sustained at the time of impact and involves local deformation and energy transformation within the cord via mechanical forces. Secondary injury is the cord response to the initial traumatic insult, developing minutes to hours post injury. Much of the process is attributed to development of ischaemia, causing axonal and neuronal necrosis and demyelination, exacerbated by decreased blood flow and loss of autoregulation.

Methylprednisolone is a synthetic glucocorticoid initially developed for anti-inflammatory actions. It was hypothesised to play a role in reducing secondary injury associated with acute spinal cord damage by limiting metabolic sequelae to which the cord is subjected. Bracken and coworkers in the NASCIS trials carried out research into use of the drug in this clinical scenario, but data obtained has recently come under intense scrutiny and been subjected to criticism. The majority of subsequent trials have failed to show any benefit of methylprednisolone.

It is currently the policy of the London Helicopter Emergency Medical Service (HEMS) to administer methylprednisolone according to a standard operating procedure. Methylprednisolone is indicated if there is no motor response to lower limbs; objective motor deficit; obvious spinal deformity; secondary signs of cord injury - priapism, diaphragmatic breathing, haemodynamic parameters (bradycardia, hypotension). These categories are associated with relevant GCS scores. A literature search was carried out to obtain evidence for and against the use of methylprednisolone in traumatic cord injury. In addition, all patients who received methylprednisolone from HEMS doctors in the last 2 years were reviewed. It is concluded that there is no good evidence to support the use of methylprednisolone as a therapeutic intervention in traumatic spinal cord injury. 